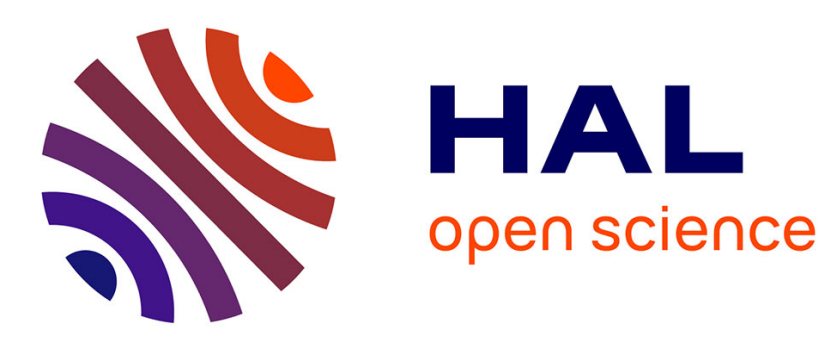

\title{
Fédéralisme et démocratie dans la Russie post-soviétique Anne Gazier
}

\section{To cite this version:}

Anne Gazier. Fédéralisme et démocratie dans la Russie post-soviétique. Revue française de droit constitutionnel, 2019, 118, pp.315-332. 10.3917/rfdc.118.0315 . hal-02364835

\section{HAL Id: hal-02364835 \\ https://hal.parisnanterre.fr/hal-02364835}

Submitted on 15 Nov 2019

HAL is a multi-disciplinary open access archive for the deposit and dissemination of scientific research documents, whether they are published or not. The documents may come from teaching and research institutions in France or abroad, or from public or private research centers.
L'archive ouverte pluridisciplinaire HAL, est destinée au dépôt et à la diffusion de documents scientifiques de niveau recherche, publiés ou non, émanant des établissements d'enseignement et de recherche français ou étrangers, des laboratoires publics ou privés. 


\section{Fédéralisme et démocratie dans la Russie post-soviétique ${ }^{1}$}

ANNE GAZIER

Fédéralisme et démocratie entretiennent des liens complexes. On peut dire, en simplifiant beaucoup, que deux grandes conceptions des rapports entre ces notions se sont succédé dans le temps. La première, retracée notamment par René Capitant ${ }^{2}$, correspond à la «théorie juridique classique » $\mathrm{du} \mathrm{XIX}^{\mathrm{e}}$ et du début du $\mathrm{XX}^{\mathrm{e}}$ siècle. Selon cette théorie, les notions de fédéralisme et de démocratie sont indépendantes l'une de l'autre car elles ne se situent pas sur le même plan : le fédéralisme concerne la forme de l'État et s'oppose à l'État unitaire tandis que la démocratie est une forme de gouvernement qui, conformément à la classification d'Aristote, se distingue de l'aristocratie et de la monarchie. Par voie de conséquence, " on peut concevoir des fédérations de monarchies, d'aristocraties ou de démocraties » et, inversement, on doit admettre que «la démocratie peut être indifféremment unitaire ou fédéraliste ». Cette conception, longtemps dominante chez les juristes et les politistes, a peu à peu cédé la place, en particulier, au $\mathrm{Xx}^{\mathrm{e}}$ siècle, aux États-Unis, à l'idée selon laquelle le fédéralisme, qui repose sur l'autonomie des entités fédérées et, qui, plus généralement, cherche à concilier l'unité et la diversité, suppose un régime qui assure le pluralisme politique, un régime démocratique $^{3}$. Pour les tenants de cette école, le fédéralisme est incompatible avec un système totalitaire ou autoritaire; il ne peut être réel que s'il est fondé sur les principes démocratiques et libéraux. Un concept a

Anne Gazier, maître de conférences HDR de droit public à l'université Paris-Nanterre, CRDP.

1. Cet article a pour origine une communication présentée lors de la Conférence internationale " Polical Transitions and Federal Projects (late $18^{\text {th }}$ - early $21^{\mathrm{st}}$ )», organisée à Paris les 24 et 25 mai 2018 par Sciences-Po Paris (Centre d'histoire de Sciences-Po), l'American University of Paris et l'université Paris-Diderot.

2. R. Capitant, "Fédéralisme et démocratie », Cours donné à la faculté de droit de Paris les 22-24 juillet 1954, Jus Politicum, ${ }^{\circ} 16$, http://juspoliticum.com/article/Federalisme-etdemocratie-1102.html Site consulté en juin 2018.

3. Il s'agissait là d'une idée dominante mais qui ne faisait pas l'unanimité, même chez les spécialistes américains du fédéralisme. En particulier, William Riker incluait dans les systèmes fédéraux ce qu'il appelait les "fédéralismes dictatoriaux ». Voir, à ce sujet, M. Burgess, A.-G. Gagnon, «Introduction », in Burgess Michael, Gagnon Alain-G., Federal Democracies, Londres, Routeledge, 2010, p. 4-6. 
même été forgé pour souligner cette identification du fédéralisme à la démocratie, celui de "federal democracy ", " démocratie fédérale " ${ }^{4}$. Cette tendance à lier étroitement fédéralisme et démocratie s'explique, notamment, sur un plan historique, par l'accroissement du nombre de pays présentant les caractéristiques d'un État tout à la fois fédéral et démocratique, dans lesquels le développement du fédéralisme s'est accompagné de l'affermissement de la démocratie libérale.

La Russie post-soviétique paraît à première vue, offrir un excellent exemple de l'étroitesse des liens entre fédéralisme et démocratie. En effet, au début des années quatre-vingt-dix, elle a entrepris de rompre avec le système soviétique unifié et centralisé et s'est lancée dans l'édification, tout à la fois, d'une démocratie libérale et d'un État fédéral. À compter des années deux mille, de façon presque symétrique, elle s'est détournée de ces deux objectifs au profit d'une recentralisation et de la mise en place d'un régime autoritaire. Ainsi, on peut estimer que la Russie post-soviétique présente le cas exemplaire d'un pays où sont allés de pair non seulement les processus de fédéralisation et de démocratisation mais aussi les processus inverses de dé-fédéralisation et de recul de la démocratie. Cependant, on peut s'interroger sur cette vision un peu simpliste. On observera d'abord que la coïncidence dans le temps des mouvements de fédéralisation et de démocratisation et de celle des mouvements opposés de mise en cause du fédéralisme et de la démocratie, n'indique pas nécessairement que les uns et les autres soient indissociables. On peut se demander, en effet, si la reconnaissance de liens aussi étroits entre ces phénomènes ne méconnaît pas tant leurs origines que leurs ressorts et, en particulier, si le "fédéralisme russe » mis en place dans la Russie post-soviétique faisait une place importante aux principes démocratiques, si son évolution a été aussi tributaire qu'il le paraît de celle des institutions politiques et si la dé-fédéralisation à laquelle on assiste aujourd'hui est liée au tournant autoritaire du régime dans son ensemble ou seulement à ses aspects centralisateurs. On peut s'interroger également sur la profondeur de ces changements : est-ce qu'ils ont atteint les bases mêmes du système fédéral russe et transformé la Russie en un État unitaire ou quasi unitaire? Ou bien est-ce qu'ils ont juste modifié le fédéralisme russe en le rendant centralisé et autoritaire?

Afin de donner des éléments de réponse à ces questions, nous étudierons la dynamique des rapports entre fédéralisme et démocratie dans la Russie post-soviétique au cours de deux périodes-clé : la période fondatrice, celle où ont été posées les bases du nouvel État russe; la période actuelle, celle des mises en cause du fédéralisme et de la démocratie.

Tout d'abord, on se demandera dans quelle mesure l'établissement d'un État fédéral en Russie, au début des années quatre-vingt-dix, a été 
lié à l'entreprise de démocratisation du système politique et si la conception du fédéralisme qui a alors prévalu, s'est appuyée ou non sur des principes démocratiques.

Ensuite, on se penchera sur l'évolution qui a conduit la Russie à s'éloigner tant de la décentralisation que de la démocratie. On recherchera dans quelle mesure la voie autoritaire suivie par le pouvoir depuis les années deux mille a mis en cause les fondements du système fédéral et comment qualifier le «fédéralisme russe » actuel.

\section{I - L'ÉDIFICATION DU « FÉDÉRALISME RUSSE » ET LA DÉMOCRATIE}

Le fédéralisme qui a été mis en place en Russie au début des années quatre-vingt-dix, trouve son origine dans l'éclatement d'une fédération d'un type bien particulier, l'Union soviétique. Rappelons à cet égard, que l'URSS était un État fédéral largement fictif. Certes, il comportait des institutions étatiques de type fédéral et une certaine protection était accordée aux langues et aux cultures des nationalités qui composaient le pays mais il s'agissait d'abord et avant tout d'un État-Parti dirigé par un parti unique et centralisé. Avec l'arrivée au pouvoir de Mikhaïl Gorbatchev, en 1985, la politique de Pereströ̈ka, vite accompagnée de la glasnost, de la transparence, a d'abord permis un développement de la liberté d'expression, l'apparition de groupes informels et l'accroissement des divisions internes du Parti communiste. Surtout, à partir de 1988, elle a entraîné la démocratisation des élections et du mode de fonctionnement des soviets, et ce à tous les niveaux territoriaux. Cette politique a eu des incidences très importantes dans les républiques fédérées où le renforcement des libertés et la tenue d'élections semi-pluralistes aux assemblées a favorisé la manifestation de revendications nationales. Ces revendications, qui visaient d'abord plus d'autonomie, se sont vite radicalisées. Elles ont conduit à l'adoption de déclarations de souveraineté puis de déclarations d'indépendance par les républiques fédérées avant d'entraîner la fin de l'URSS's.

Cette évolution a eu des répercussions au sein même de la principale république fédérée, la RSFSR (République socialiste fédérative soviétique de Russie). On précisera que cette république, qualifiée officiellement de "fédérative " était une fédération encore plus fictive que l'URSS. Elle comprenait, au niveau régional, deux types d'entités : des

5. Sur cette évolution, voir notamment A. Gazier, » Deuxième partie. Le bouleversement des institutions et de la vie politique », in Colas Dominique (dir.), L'Europe post-communiste, Puf, p. 133 sq. 
régions appelées «oblasts» ou «kraïs », dénuées de toute autonomie ; des formations - dénommées districts (okrougs), oblasts et républiques - qualifiées d' « autonomes ». Ces dernières avaient la particularité d'être constituées sur une base ethnique et de comprendre des représentants d'une ou plusieurs "nationalités » autres que russe. Elles seules se voyaient appliquer - de façon purement formelle - certains principes fédéralistes. En particulier, les républiques autonomes avaient leur propre constitution et des institutions calquées sur celles de la RSFSR (comprenant un Soviet suprême et un Conseil des ministres). Cependant, elles étaient placées, dans les faits sinon en droit, sur un pied d'égalité avec les autres entités régionales. Le processus d'éclatement de l'URSS a conduit les républiques autonomes à prendre modèle sur leurs « grandes sœurs », les républiques fédérées, à adopter, tout comme elles, des déclarations de souveraineté. Certes, à l'exception de la Tchétchénie et du Tatarstan, elles n'avaient pas de visées indépendantistes et s'efforçaient surtout d'accroître leur autonomie aussi bien politique qu'économique tout en restant au sein de la Russie. Malgré ces limites, ce phénomène a provoqué la réaction des autres entités régionales qui ont cherché à renforcer leurs propres droits et, surtout, à préserver l'unité de la Russie, et qui ont réclamé, à cette fin, la création d'un véritable État fédéral. C'est cet enchaînement d'événements qui est à l'origine de la création de la Fédération de Russie suite à la fin de l'URSS, en $1991^{6}$.

Comme on peut le constater, la politique de démocratisation menée sous la Perestroìka a joué un rôle décisif dans l'évolution qui a débouché sur l'adoption d'un système fédéral par le nouvel État russe. Faut-il en déduire que le processus de démocratisation a profondément marqué celui de fédéralisation? Nous rechercherons dans un premier temps quelles incidences a eu la démocratisation des institutions politiques sur l'édification d'un État fédéral, puis dans quelle mesure les idées démocratiques ont influé sur les caractéristiques du fédéralisme mis en place.

\section{A - LA DÉMOCRATISATION DU SYSTÈME POLITIQUE ET L'INSTAURATION D'UN SYSTÈME FÉDÉRAL}

Comme nous l'avons signalé, la Perestroïka a lancé toute une série de mesures visant à démocratiser, de haut en bas, l'ensemble du système étatique, comportant la tenue d'élections semi-libres aux soviets et un renforcement de leurs pouvoirs au détriment de ceux des comités exécutifs et des organes du Parti communiste. Ces mesures ont été accompagnées d'une politique de décentralisation au profit des soviets locaux ${ }^{7}$.

6. Voir ibid., p. 165 sq.

7. Voir notamment, sur cette politique de décentralisation, A. Gazier, «La Russie à la recherche d'un système local conciliant démocratie et efficacité », Problèmes politiques et sociaux, $\mathrm{n}^{\circ} 683$, La documentation française, juin 1992, p. 4 sq. 
Au sein de la RSFSR, cette réforme a permis notamment que le pluralisme fasse son apparition dans l'ensemble des assemblées locales. Cependant, elle n'a pas été suivie des mêmes conséquences dans toutes les entités régionales. Dans les républiques autonomes, elle a favorisé le processus d'émancipation politique de ces entités, qui les a conduites, en particulier, à adopter de nouvelles constitutions et à faire un usage intensif de leur pouvoir législatif. En revanche, dans les autres entités régionales, elle n'a pas entraîné le développement d'une telle autonomie, en raison du maintien de liens étroits avec le centre. Il en résultera une inégalité qui sera consacrée dans un texte essentiel pour l'avenir du fédéralisme russe, le "Traité fédéral » en date du 31 mars 1992, conclu par le pouvoir central avec la quasi-totalité des entités régionales, appelées «sujets de la Fédération ${ }^{8}$. Ce texte prévoyait, en effet, l'établissement d'un système fédéral asymétrique, donnant aux républiques des droits nettement plus étendus qu'aux autres membres de la Fédération ${ }^{9}$.

Sur un plan institutionnel, tout particulièrement, la politique de démocratisation n'a pas eu les mêmes incidences dans les républiques et dans les autres entités régionales. En effet, les républiques, qui ont pu choisir librement leurs institutions, se sont toutes dotées d'un exécutif élu, la plupart du temps au suffrage universel direct, alors que les autres unités régionales se sont vues imposer par le centre un organe exécutif élu par le soviet puis, à compter d'août 1991, un chef de l'administration régionale - vite appelé «gouverneur » - nommé par le président de la RSFSR. Il ne faudrait pas se méprendre sur la signification de cette différence. Si presque toutes les républiques ont choisi d'avoir un Président élu au suffrage universel direct, ce n'est pas tant pour rendre leurs institutions plus démocratiques que pour suivre l'exemple du président de la RSFSR élu directement par le peuple depuis juin 1991, pour avoir un exécutif fort et lui permettre d'avoir plus de légitimité et de poids face au centre ${ }^{10}$. Quant à la nomination des gouverneurs dans les régions, elle avait été décidée à la suite du putsch d'août 1991 et avait vocation à n'être que provisoire. Cependant, cette mesure, éminemment

8. Ce traité était formé de trois accords distincts, conclus avec les trois catégories de «sujets de la Fédération»: les formations dites nationales-étatiques : les républiques (à l'exception du Tatarstan et de la République tchétchène-ingouche); les formations dites nationales-territoriales : l'oblast autonome et les districts autonomes; les formations dites administratives-territoriales : les kraïs, les oblasts et les villes de Moscou et SaintPétersbourg.

9. Voir notamment, sur le traité fédéral et sur l'évolution des rapports entre le centre et les régions durant cette période clé, C. Ross, Federalism and democratization in Russia, Manchester, Manchester University Press, 2002, tout particulièrement p. 17 sq.

10. Voir, à titre d'exemple, V.A. Vodjanitskij, «Stanovlenie instituta prezidentsva v respublike Saha (Iakutija) (L'établissement de l'institution présidentielle dans la république de Sakha (Iakoutie)) », Istorieskaja i social'no-obrazovatel'naja mysl', no 5 (15), 2012, p. 103-108. 
centralisatrice, qui visait, selon l'expression employée à l'époque, à renforcer la « verticale exécutive » s'est appliquée pendant plusieurs années, jusqu'en 1995-96. Ainsi, durant les années où le système étatique russe s'est construit, les républiques (qui ne couvraient que 28,6\% de la superficie de la Russie et ne comprenaient que $15,2 \%$ de sa population) avaient à leur tête des exécutifs élus tandis que les autres entités régionales étaient dirigées, sauf exceptions, par des gouverneurs nommés. Si on ajoute que Boris Eltsine a entrepris, toujours à compter d'août 1991, de désigner également, dans les oblasts et les kraïs, des représentants du Président chargés d'une mission d'information et de contrôle, on mesure à quel point les dirigeants de ces régions étaient liés au centre et échappaient à tout contrôle démocratique.

Ainsi, la démocratisation lancée par la Pereströ̈ka, assortie d'une politique de décentralisation, a indéniablement favorisé le processus de fédéralisation de la Russie. Mais cette démocratisation a aussi été limitée par l'émergence d'un fédéralisme à deux vitesses, d'un « fédéralisme asymétrique $^{11} »$. Par la suite, la Constitution de 1993 va proclamer l'égalité entre l'ensemble des sujets de la Fédération et atténuer quelque peu la différence de statut entre les républiques et les autres sujets mais sans la supprimer totalement. Ce changement a-t-il été accompagné d'une meilleure protection de l'autonomie et de la démocratie dans les membres de la Fédération ${ }^{12}$ ?

\section{B - LA CONSTITUTION DE 1993 : LES FONDEMENTS AMBIGUS D'UN ÉTAT FÉDÉRAL ET DÉMOCRATIQUE}

Selon l'article $1^{\text {er }}$ de la Constitution de 1993, la Russie est « un État de droit, démocratique, fédéral, ayant une forme républicaine de gouvernement ». À première vue, les normes constitutionnelles établissent un État conforme tout à la fois aux standards d'une démocratie libérale et aux principes d'un État fédéral. S'agissant du premier trait, on reprendra la définition de la démocratie libérale donnée par Philippe Ardant et Bertrand Mathieu, selon laquelle, il s'agit d'un «système qui met en ouvre deux principes : le principe démocratique - qui renvoie à la souveraineté du peuple, comme instrument de la légitimation du pouvoir - et le principe libéral - qui implique la limitation du pouvoir

11. Voir notamment, C. Ross, Federalism and democratization in Russia, op. cit., p. 175 sq.

12. La Russie comptait, en 1993, 89 sujets de la Fédération : 21 républiques, un oblast autonome, 10 districts autonomes, 6 kraïs, 49 oblasts, 2 villes d'importance fédérale (Moscou et Saint-Pétersbourg). Suite à la fusion de plusieurs sujets de la Fédération (entre 2003 et 2008) et à l'annexion de la Crimée, en 2014 - qui s'est traduite par la création de deux nouveaux sujets : la république de Crimée et la ville d'importance fédérale de Sébastopol - on dénombre 85 sujets de la Fédération : vingt-deux Républiques, un oblast autonome, 4 districts autonomes, 9 kraïs, 46 oblasts et 3 villes d'importance fédérale. 
(séparation des pouvoirs et droits fondamentaux) $)^{13}$ ». La Constitution russe de 1993 semble pleinement adhérer à cette conception de la démocratie. En effet, elle proclame, notamment dans son premier chapitre, l'ensemble des principes énoncés ci-dessus - souveraineté du peuple, pluralisme politique, séparation des pouvoirs - et comprend, dans son deuxième chapitre, une longue et précise énumération des droits et libertés, conforme aux normes internationales en la matière et, tout particulièrement, à la Convention européenne des droits de l'homme ${ }^{14}$. En outre, les dispositions de ces deux chapitres font l'objet d'une protection particulière car elles sont presque impossibles à réviser (à moins d'adopter une nouvelle Constitution). Relevons également que la Commission de Venise (Commission européenne pour la démocratie par le droit qui dépend du Conseil de l'Europe) a, dans l'avis qu'elle a émis sur la Constitution de 1993, estimé que ce texte "ne présente pas de problème important de non-conformité aux principes d'un État démocratique fondé sur le droit et respectueux des droits de l'homme ${ }^{15}$ ».

De même, la Constitution prévoit un système fédéral qui répond grosso modo aux grandes « lois » du fédéralisme qui ont été dégagées par Georges Scelle : superposition des ordres constitutionnels et juridiques de la Fédération et des États fédérés; autonomie des États fédérés et participation de ces derniers à la formation de la « volonté fédérale » par le biais d'une Chambre haute ${ }^{16}$. Plus précisément, la Constitution russe établit une superposition de l'ordre constitutionnel et juridique de la Fédération et de celui de chacun de ses sujets, une délimitation des compétences de la Fédération et de celles des sujets de la Fédération, la primauté du droit fédéral sur celui des composantes de la Fédération. Elle reconnaît également que les sujets de la Fédération sont chacun

13. Ph. Ardant, B. Mathieu, Droit constitutionnel et institutions politiques, Issy-Les-Moulineaux, LGDJ, Lextenso, 29e éd., 2017-2018, p. 164.

14. Voir notamment S. Belov, « Russia : Foreign Transplants in the Russian Constitution and Invisible Foreign Precedents in Decisions of the Russian Constitutional Court », in Groppi Tania, Ponthoreau, Marie-Claire (dir.), The Use of Foreign Precedents by Constitutional Judges, Oxford and Portand, Oregon, Hart Publishing, 2013, p. 347-341 ; P. Gélard, "L'actualité constitutionnelle en Russie (novembre 1993-janvier 1994) », Revue française de droit constitutionnelle, $\mathrm{n}^{\circ} 17,1994$, p. 188-189.

15. Voir «Avis sur la Constitution de la Fédération de Russie adoptée en votation populaire le 12 décembre 1993 », CDL (1994)011f-resr, Strasbourg, 16 mars 1994.

16. Voir notamment M. Croisat, Le fédéralisme dans les démocraties contemporaines, Montchrestien, $2^{\mathrm{e}}$ éd., 1995 , p. 25 . L'auteur évoque ici, comme première loi, le "principe de séparation " mais la plupart des auteurs qui font référence aux principes du fédéralisme dégagés par Georges Scelle préferent l'expression " principe de superposition ", plus évocatrice. Citons, à titre d'exemples, L. Favoreu, P. Gaïa, R. Ghevontian, J.-L. Mestre, O. Pfersmann, G. Scoffoni, A. Roux, Droit constitutionnel, Dalloz, $20^{\mathrm{e}}$ éd., septembre 2017, p. $470 \mathrm{sq}$.; J. Gicquel et J.-E. Gicquel, Droit constitutionnel et institutions politiques, LGDJ, Lextenso, $31^{\mathrm{e}}$ éd., 2017-2018, p. 94 sq. 
dotés d'une constitution (ou d'un «statut $)^{17}$ et de leurs propres organes législatifs et exécutifs. Enfin, elle prévoit la représentation de chacun des sujets de la Fédération au sein de la Chambre haute du Parlement fédéral, le Conseil de la Fédération. Il convient d'ajouter que le système étatique satisfait à un autre critère du fédéralisme, posé par certains auteurs comme Ferran Requejo ${ }^{18}$ - celui de comporter un «arbitre institutionnel » (qui soit une juridiction suprême). En effet, la Constitution russe institue une Cour constitutionnelle fédérale chargée, entre autres, de se prononcer sur les conflits de compétence entre la Fédération et ses composantes.

Cependant, une lecture plus attentive des dispositions constitutionnelles conduit à nuancer ces affirmations. Tout d'abord, la Constitution de 1993 établit un système politique de type présidentialiste, qui confere au chef de l'exécutif des pouvoirs très étendus, ce qui favorise la personnalisation du pouvoir, déséquilibre les institutions politiques et n'est guère propice à leur fonctionnement démocratique ${ }^{19}$. Ensuite, le texte constitutionnel consacre à l'article $5 \S 3$ un principe hérité de la période soviétique, le "principe de l'unité du pouvoir d'État». Ce principe, qui se rattache à une conception hiérarchique des relations entre le centre et la périphérie, signifiait, dans les textes soviétiques, la stricte subordination des organes inférieurs aux organes supérieurs. Certes, dans la Constitution de 1993, ses implications sont moindres. Elles se limitent aux rapports entre les organes exécutifs de la Fédération et ceux de ses sujets. Notamment, l'article $77 \S 2$ dispose que les instances exécutives fédérales et celles des sujets de la Fédération «forment un système unique du pouvoir exécutif ». À cette disposition vague s'ajoute un pouvoir précis, donné au Président de la Fédération, celui de suspendre, en cas d'illégalité, les actes des organes exécutifs des sujets de la Fédération (article $85 \S 2$ de la Constitution). Même s'il ne signifie plus une centralisation de type soviétique, ce principe vient donc sérieusement limiter l'autonomie reconnue aux composantes de la Fédération. Par exemple, en matière institutionnelle, il est prévu, à l'article $77 \S 1$, que les sujets de la Fédération établissent «de façon autonome » leurs institutions mais cette liberté est strictement limitée non seulement par les «fondements de l'ordre constitutionnel » et par une loi fédérale fixant les normes essentielles en la matière mais aussi par le principe de l'unité du

17. Les républiques sont dotées d'une "constitution " tandis que les autres entités régionales n'ont qu'un simple «statut » mais cette différence a un caractère formel et n'entraîne pas de conséquence juridique.

18. Voir les principes du fédéralisme dégagés par Ferran Requejo, cités par C. Ross "Federalism and defederalisation in Russia », in Gill Graeme, Young James, (dir.), Routledge Handbook of Russian Politics and Society, Londres, New York, Routledge, 2012, p. 140.

19. Voir notamment, J.-P. Massias, Droit constitutionnel des États d'Europe de l'Est, Puf,

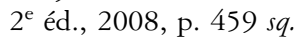


pouvoir d'État. La Cour constitutionnelle de la Fédération de Russie a fait une large utilisation de ce principe, notamment pour restreindre la liberté des sujets de la Fédération dans le choix de leurs institutions. En particulier, dans deux arrêts célèbres, datant de 1996, la Cour s'est fondée sur ce principe pour imposer une sorte de "mimétisme institutionnel » en censurant des normes du statut du kraï de l'Altaï et de celui de l'oblast de Tchita qui prévoyaient l'élection des gouverneurs par l'assemblée régionale ${ }^{20}$. En effet, la Cour a estimé que l'unité du pouvoir d'État exigeait que les sujets de la Fédération reproduisent à leur niveau, le mode d'organisation du pouvoir fédéral et donc que les gouverneurs soient élus au suffrage universel direct ${ }^{21}$.

Il convient aussi de signaler que la Constitution comprend un autre élément centralisateur qui vient se heurter aux principes du fédéralisme : elle prévoit un système judiciaire centralisé, les juridictions relevant de la Fédération et non de ses sujets ${ }^{22}$.

Enfin, on observera que la Constitution contient des dispositions contradictoires ou, tout au moins, difficiles à concilier. Il en est ainsi des normes relatives à la répartition des compétences. D'une part, les articles 71 à 73 énumèrent les matières relevant de la compétence exclusive de la Fédération puis de la compétence conjointe de la Fédération et de ses sujets et reconnaissent à ces derniers une compétence de droit commun pour ce qui reste, sans faire aucune distinction entre les différentes catégories de sujets. D'autre part, l'article $11 \S 3$ dispose que la répartition des compétences entre la Fédération et ses sujets ne résulte pas seulement de la Constitution mais aussi du Traité fédéral et d'autres " traités de délimitation des domaines de compétence et des attributions ». Cette dernière disposition a fait l'objet d'une interprétation large sous Boris Eltsine (qui a conclu des traités bilatéraux avec une quarantaine de sujets de la Fédération entre 1994 et 1999, souvent en contradiction avec les articles 71 à 73 ). Il en résultera un "fédéralisme contractuel » ou un «fédéralisme à la carte » entrant en conflit tant avec la répartition constitutionnelle des compétences qu'avec le principe de l'égalité des sujets de la Fédération ${ }^{23}$.

20. Arrêt de la Cour constitutionnelle de la Fédération de Russie du 18 janvier 1996 «Sur l'examen de la constitutionnalité de plusieurs dispositions du Statut (de la Loi fondamentale) du kraï de l'Altaï », Sobranie zakonodatel'stva Rossijskoj Federacii, 1996, no 4, art. 409 ; arrêt de la Cour constitutionnelle de la Fédération de Russie du $1^{\text {er }}$ février 1996 «Sur l'examen de la constitutionnalité de plusieurs dispositions du Statut (de la Loi fondamentale) de l'oblast de Tchita ", Sobranie zakonodatel'stva Rossijskoj Federacii, 1996, $\mathrm{n}^{\circ}$ 7, art. 700 .

21. Voir, à ce sujet, A. Gazier, "Justice constitutionnelle et fédéralisme en Russie ", Revue du droit public, $\mathrm{n}^{\circ}$ 5, 1999, p. 1385 sq.

22. Il convient de préciser qu'il y a des exceptions à ce principe (qui ne sont pas prévues par la Constitution) : certaines juridictions - les juges de paix et les juridictions constitutionnelles des sujets de la Fédération - ne dépendent pas de la Fédération mais de ses sujets.

23. Sur ce "fédéralisme contractuel", voir notamment A. Gazier, « La contractualisation des rapports entre le centre et les régions et l'édification du fédéralisme en Russie ", in 
Le texte constitutionnel contient donc des dispositions qui limitent ou affaiblissent aussi bien le caractère démocratique des institutions que la nature fédérale de l'État. Il serait ainsi abusif d'estimer que la Constitution aurait posé les bases solides tant d'une démocratie libérale que d'un État fédéral et que ce serait la pratique qui aurait conduit la Russie à s'éloigner de cet idéal démocratique et fédéral. En effet, les dérives autoritaires et clientélistes qui se sont développées dans les années quatre-vingt-dix ne sont pas toutes intervenues en violation ou en marge de la Constitution mais se sont bien souvent appuyées sur l'interprétation de dispositions constitutionnelles ambiguës (comme le principe de l'unité du pouvoir d'État ou la possibilité de définir contractuellement les compétences régionales). Cependant, il est vrai qu'un fossé s'est vite creusé entre le droit et le fait. En particulier, si, comme on l'a vu, les républiques de la Russie se sont dotées d'institutions démocratiques et, souvent, d'un chef de l'exécutif élu au suffrage universel direct, elles ont instauré le plus souvent, dans les faits, un régime autoritaire et créé de véritables fiefs régionaux. Ainsi, l'autonomie qui leur a été conférée n'a pas servi à renforcer la démocratie mais à installer durablement au pouvoir une élite tant politique qu'économique ${ }^{24}$. D’une façon générale, les années Eltsine ont été marquées par la montée en puissance des dirigeants régionaux, par les grandes libertés prises par ces derniers avec le droit fédéral, et par la relative faiblesse du pouvoir central.

Au total, la période fondatrice du nouvel État russe ne semble pas avoir été caractérisée par une véritable synergie entre démocratisation et fédéralisation. Les liens entre ces deux processus ont été plus complexes. En particulier, comme l'affirme Cameron Ross après avoir étudié les pouvoirs régionaux sous Eltsine, «plus d'autonomie ne conduit pas nécessairement à plus de démocratie ${ }^{25} »$. Allant plus loin, on peut estimer que dans les années quatre-vingt-dix, le développement d'un fédéralisme asymétrique et à la carte non seulement n'a pas favorisé mais encore a gêné la démocratisation dans le pays ${ }^{26}$.

Malgré tout, il convient de reconnaître que, durant ces années, une structure fédérale a été mise en place en Russie tandis que certains éléments clé d'un système démocratique libéral (comme le pluralisme politique, les élections libres, la protection des libertés fondamentales) ont été établis par les textes et introduits, au moins en partie, dans les pratiques.

Fortin Yvonne (dir.), La contractualisation dans le secteur public des pays industrialisés depuis 1990, L'Harmattan, 1999, p. 329 sq.

24. Voir notamment C. Ross « Federalism and defederalisation in Russia », op. cit., p. 141.

25. Idem.

26. Voir notamment N. Douglas, «"De-federalisation” vs. democratisation. The politicisation of federal reforms in Russia ", Federal Governance, vol. 10, n ${ }^{\circ}$ 2, p. 29-30 ; R. Sakwa, "Federalism and democracy in the Russian Federation ", in Burgess Michael, Gagnon Alain-G, Federal Democracies, op. cit., p. 211. 
Avec l'arrivée au pouvoir de Vladimir Poutine, une politique centralisatrice et de plus en plus autoritaire va changer la donne en s'attaquant aux institutions de la démocratie libérale ainsi qu’à la forme fédérale de la Russie.

\section{II - LE « FÉDÉRALISME RUSSE » ET LA RECENTRALISATION AUTORITAIRE}

Depuis le début des années deux mille, Vladimir Poutine a mené deux politiques complémentaires qui ont permis de mieux asseoir son pouvoir : la restriction du pluralisme politique et la recentralisation de l'État. La première a consisté à multiplier les barrières mises à l'entrée dans la compétition électorale et politique, à favoriser la montée en puissance d'un véritable parti du pouvoir, le parti «Russie Unie », et à encadrer strictement la société civile. S'y sont ajoutées peu à peu, surtout dans les années 2010, des limitations apportées aux principales libertés des citoyens (liberté d'association, de manifestation, d'expression, etc.). La politique de recentralisation, quant à elle, a été lancée peu après l'arrivée au pouvoir de Vladimir Poutine. Elle visait à mettre un terme à une situation marquée par la faiblesse du pouvoir central face aux régions. Il s'agissait de lutter contre les véritables féodalités régionales qui s'étaient constituées et contre certains effets de l'accroissement de l'autonomie des régions (comme la prolifération d'actes contraires à la Constitution et aux lois fédérales et l'emprise croissante des autorités régionales sur les organes territoriaux de la Fédération). L’idée était aussi de mettre un terme au fédéralisme asymétrique et «à la carte » qui s'était développé sous Boris Eltsine.

Comme on le voit, la politique de recentralisation visait, au moins au début, à rétablir l'ordre fédéral et un fonctionnement plus équilibré de la Fédération, notamment en assurant la primauté du droit fédéral sur le droit des sujets de la Fédération. Ce faisant, elle permettait de revenir sur les libertés prises par les autorités régionales dans tous les domaines, y compris les droits et libertés, et allait plutôt dans le sens d'un renforcement du rôle du droit et de l'édification du fédéralisme ${ }^{27}$. Toutefois, la poursuite de cette politique, assortie des restrictions apportées au pluralisme politique, a peu à peu atteint les bases mêmes du fédéralisme, au point que l'on peut se demander si la Russie présente encore les traits d'un État fédéral.

27. Voir notamment N. Douglas, "De-federalization” vs. Democratization. The Politisation of Federal Reforms in Russia », ibid., p. 29 ; R. Sakwa, "Federalism and democracy in the Russian Federation », ibid., p. 218. 


\section{A - LA MISE EN CAUSE DES BASES DU FÉDÉRALISME}

Si on reprend les grands principes du fédéralisme énumérés précédemment (superposition, autonomie, participation), c'est le deuxième d'entre eux, le principe de l'autonomie des entités fédérées qui a été atteint le plus sévèrement par la politique de recentralisation menée à compter de 2000. Dès le premier mandat de Vladimir Poutine, tout un ensemble de mesures - l'instauration de représentants du Président dans des super régions (les districts fédéraux) englobant plusieurs sujets de la Fédération, l'affaiblissement du statut des dirigeants régionaux (grâce à leur éviction de la Chambre haute, le Conseil de la Fédération, et à leur soumission à une tutelle présidentielle) ainsi qu'une sévère limitation des compétences régionales - a considérablement diminué l'autonomie des sujets de la Fédération et a conduit à l'instauration d'un fédéralisme très centralisé 28 .

Mais il faudra attendre fin 2004 pour qu'un changement ébranle véritablement les fondements d'un État fédéral. Ce changement a consisté à bouleverser le mode de désignation et de révocation des chefs des exécutifs des sujets de la Fédération en les plaçant dans un rapport de dépendance étroit avec le président de la Russie. Un bref retour en arrière s'impose : après une période marquée par l'existence d'une différence de situation entre les présidents des républiques, élus par la population (directement ou indirectement) et les gouverneurs des autres entités régionales, nommés par le président de la Russie, il a été décidé, fin 1995, que ces derniers seraient élus au suffrage universel direct. Ainsi, à compter de 1996, tous les chefs des exécutifs régionaux ont été élus directement par le peuple (si l'on excepte les dirigeants de quelques républiques, élus par leur assemblée législative). Or, une loi du 11 décembre $2004^{29}$, prise pour renforcer «la verticale du pouvoir », a mis fin à ce mode d'élection pour tous les chefs des exécutifs régionaux (y compris les présidents des républiques). Elle lui a substitué une élection par l'assemblée régionale sur proposition du président de la Russie, ce qui revenait à une nomination de fait par le chef de l'État (d'autant plus que si l'assemblée s'opposait plus de deux fois de suite au choix du Président, ce dernier pouvait la dissoudre). En outre, cette loi a rendu

28. Voir notamment, sur ces réformes centralisatrices, A. Gazier, «La mise au pas des régions russes? La réforme institutionnelle de Vladimir Poutine ", Le courrier des pays de l'Est, $\mathrm{n}^{\circ}$ 1015, mai 2001, p. 4 sq.; J. Radvanyi, «Le fédéralisme russe : des mutations inachevées ", Regards de l'Observatoire franco-russe, Le Cherche-Midi, 2013. <hal-01436160>.

29. Loi de la Fédération de Russie du 11 décembre 2004 ( $\mathrm{n}^{\circ}$ 159-FZ) Sur l'amendement de la loi fédérale "sur les principes généraux d'organisation des organes législatifs (représentatifs) et exécutifs des sujets de la Fédération de Russie " et de la loi fédérale "Sur les garanties essentielles des droits électoraux et du droit des citoyens de la Fédération de Russie de participer à un référendum ", Sobranie zakonodatel'stva rossijskoj Federacii, 2004, $\mathrm{n}^{\circ} 50$, art. 4950 . 
possible la révocation discrétionnaire des chefs des exécutifs régionaux par le Président.

À l'appui d'une telle mise sous tutelle a été invoqué, entre autres, le principe constitutionnel de l'unité du pouvoir d'État. La Cour constitutionnelle, saisie de la question de la constitutionnalité de la loi de 2004, a reconnu celle-ci conforme à la Constitution ${ }^{30}$, en se fondant notamment sur une interprétation pour le moins extensive de ce principe. Elle a ainsi déduit de l'unité du pouvoir d'État et du «système unique du pouvoir exécutif » qui en découle, l'existence d'un rapport « de subordination directe » des chefs des exécutifs régionaux à l'égard du président de la Russie. À l'inverse, la Cour a donné de l'autonomie des sujets de la Fédération dans le choix de leurs institutions une portée très limitée, soulignant qu'elle était strictement encadrée par la législation fédérale ${ }^{31}$.

Surtout, cette nouvelle procédure de désignation des exécutifs régionaux venait se heurter aux bases d'un État fédéral. Certes, l'organisation fédérale d'un État n'impose pas l'élection au suffrage universel direct des exécutifs des entités fédérées. Toutefois, en confiant le choix des dirigeants régionaux au président de la Russie, la loi de 2004 allait directement à l'encontre d'un des principes essentiels du fédéralisme, le principe qui veut que les organes des entités fédérées soient « formés par ces dernières de façon autonome, sans ingérence du niveau fédéral ${ }^{32}$ ". En effet, dans un État fédéral, la légitimité de l'exécutif des entités fédérées ne saurait procéder du pouvoir fédéral ${ }^{33}$. S'il existe, malgré tout, des Fédérations, comme l'Union indienne, où les gouverneurs des États sont nommés par le Président de l'Union, il s'agit de rares exceptions.

30. Voir l'arrêt de la Cour constitutionnelle de la Fédération de Russie du 21 décembre $2005\left(\mathrm{n}^{\circ} 13-\mathrm{P}\right)$ «Sur les principes généraux d'organisation des organes législatifs (représentatifs) et exécutifs du pouvoir d'État des sujets de la Fédération de Russie suite aux plaintes de plusieurs citoyens ", Sobranie zakonodatel'stva rossijskoj Federacii, 2006, no 3, art. 336.

31. On observera que la Cour a été amenée à opérer un revirement de jurisprudence dans cet arrêt. En effet, comme on l'a vu, elle avait jugé, dans les arrêts de 1996 relatifs aux statuts du kraï de l'Altaï et de l'oblast de Tchita, que le principe de l'unité du pouvoir d'État imposait que les gouverneurs soient élus au suffrage universel direct. Or elle a estimé qu'elle devait revenir sur cette solution et opérer un revirement pour tenir compte des « conditions sociojuridiques » de l'application des normes constitutionnelles. Voir, notamment, sur l'arrêt du 21 décembre 2005, M.-E. Baudoin, «La réception des normes européennes : le juge constitutionnel russe et la Convention européenne des droits de l'homme », Revue d'études politiques et constitutionnelles Est-européennes, 2008, $\mathrm{n}^{\circ} 2$ (CEI), p. 98 sq. ; A. Gazier, «Le Président de la Fédération de Russie et l'instauration d'une «verticale exécutive », Revue d'études comparatives Est-Ouest, 2008, vol. 39, n 2, p. 74 sq.

32. Voir notamment l'avis de la Commission de Venise à ce sujet, Avis relatif au projet de loi fédérale sur les modifications à apporter à la loi fédérale «Sur les grands principes d'organisation des organes législatifs (représentatifs) et exécutifs des sujets de la Fédération de Russie » et à la loi fédérale «Sur les garanties essentielles des droits électoraux et du droit de participation au référendum des citoyens de la Fédération de Russie ", $\mathrm{N}^{\circ} 321$ / 2004, 6 décembre 2004, CDL-AD(2004)042, p. 7.

33. Voir notamment G. Marcou, «Fédéralisme et centralisation en Fédération de Russie : le statut des gouverneurs des sujets de la Fédération ", in L'État et le droit d'est en ouest. Mélanges offerts au Professeur Michel Lesage, Société de législation comparée, 2006, p. 482 sq. 
Un tel changement a fait l'objet de critiques par des instances comme le Congrès des pouvoirs locaux et régionaux du Conseil de l'Europe ou la Commission de Venise, non seulement pour sa mise en cause du fédéralisme mais aussi pour son caractère antidémocratique car les électeurs se sont vus privés de la possibilité de choisir directement et librement les chefs des exécutifs régionaux ${ }^{34}$. On peut dire que, dans ce cas de figure, le recul du fédéralisme a été véritablement de pair avec celui de la démocratie. Cette mesure anti-fédérale a d'ailleurs surtout été critiquée, en Russie même, pour être revenue sur ce qui était considéré comme un acquis démocratique, l'élection des gouverneurs par le peuple.

La loi de 2004 a marqué un véritable tournant dans l'évolution du fédéralisme russe, en changeant pour longtemps la nature des liens entre le président et les exécutifs régionaux. Certes, suite aux grandes manifestations de protestation de 2011-2012, l'élection au suffrage universel direct des chefs des exécutifs régionaux a été réintroduite. Cependant, ce changement a été limité par l'instauration d'un système de filtrage (permettant d'écarter les candidats indésirables) ${ }^{35}$ puis, un an plus tard, par la possibilité donnée aux sujets de la Fédération q i le souhaitent, d'en revenir à l'élection de leur dirigeant par l'assemblée sur proposition du président ${ }^{36}$. En outre, se sont développées des pratiques qui sont venues restreindre encore la liberté des électeurs comme la démission de gouverneurs sous l'impulsion du chef de l'État, la nomination à leur place de gouverneurs par intérim et la ratification du choix de ces derniers lors des élections ${ }^{37}$.

Un autre principe essentiel du fédéralisme a été mis à mal par la politique centralisatrice de Vladimir Poutine, celui de la participation des

34. Voir notamment, Congrès des pouvoirs locaux et régionaux du Conseil de l'Europe, Recommandation 297 (2010), La démocratie locale et régionale en Fédération de Russie, et, tout particulièrement le point 5 en invitant les autorités russes «à réintroduire des élections directes pour les gouverneurs de région afin de restaurer le niveau de démocratie régionale en vigueur en Fédération de Russie avant 2004 »; A.K. Stahl, "Russia’s Path to Federalism and Democracy under the Influence of the Council of Europe ", L'Europe en formation, 2011/1 ( $\left.\mathrm{n}^{\circ} 359\right)$, p. $70 \mathrm{sq}$.

35. La loi du 2 mai 2012 ( $\mathrm{n}^{\circ}$ 40-FZ) (Rossijskaja gazeta, 4 mai 2012) qui a réintroduit le système de l'élection au suffrage universel direct des chefs des exécutifs régionaux, a institué, en effet, un système complexe de sélection des candidats. L'élément principal de ce système est ce que l'on appelé un «filtre municipal », chaque candidat devant obtenir le soutien de 5 à $10 \%$ des élus municipaux pour pouvoir se présenter à l'élection du gouverneur.

36. Ce choix, permis par une loi du 2 avril 2013 ( ${ }^{\circ}$ 30-FZ) (Rossijskaja gazeta, 5 avril 2013), a été fait, pour le moment, par neuf sujets de la Fédération. Voir, au sujet de la réforme de 2012-2013, R.S. Muhametov, « evolljucija sistemy izbranija glav regionov v Rossii »(L'évolution du système de désignation des chefs des régions en Russie), Filosofija politiki i politologija, 2016, p. 80 sq.

37. Voir notamment V. Shtepa, "Kemlin Uses "Preventive Democracy" to Reinforce Russia's Post-Fedralism (Part One) », 17 novembre 2017 https://jamestown.org/program/ kremlin-uses-preventive-democracy-reinforce-russias-post-federalism-part-two/ Site consulté en mai 2018. 
entités fédérées à la formation de la volonté fédérale par le biais d'une Chambre haute assurant leur représentation. En conformité avec ce principe, la Constitution prévoyait, dans sa rédaction initiale, que la Chambre haute du Parlement, le Conseil de la Fédération, comprenait un représentant de l'organe représentatif et un représentant de l'organe exécutif de chacun des sujets de la Fédération, renvoyant à la loi le soin de préciser les modalités de désignation de ces membres. Or, plusieurs changements sont intervenus dans la composition de cette assemblée et ont porté atteinte à sa fonction de représentation des régions. En simplifiant beaucoup, on peut dire que, de 1995 à 2000, les dirigeants des régions siégeaient de plein droit au Conseil de la Fédération, ce qui était propice à la défense des intérêts régionaux. En 2000, il a été décidé d'évincer ces responsables de la Chambre haute et de confier la désignation des « sénateurs » aux organes dirigeants des sujets de la Fédération, ce qui a affaibli leur statut. Puis, en 2004, le changement du mode de désignation des gouverneurs qui est intervenu, n'a pas manqué d'avoir des répercussions sur la Haute assemblée, dont la moitié des membres ont vu leur nomination confiée à des chefs des exécutifs régionaux eux-mêmes choisis par le président de la Russie. Il en est résulté non seulement une diminution du rôle joué par le Conseil de la Fédération dans la représentation des régions mais aussi une atteinte - indirecte mais importante - au principe de séparation des pouvoirs.

Un pas supplémentaire a été franchi en 2014, lors u'une révision constitutionnelle (en date du 21 juillet), a permis au président de la Russie de nommer lui-même des «représentants de la Fédération » au sein de la Chambre haute. Certes, il est prévu que ces nouveaux membres doivent s'ajouter à ceux qui sont désignés par les sujets de la Fédération et ne pas dépasser $1 / 10^{\mathrm{e}}$ de leur nombre. Mais cette possibilité offerte au président de la Fédération de Russie de désigner un nombre non négligeable de sénateurs (jusqu'à 17 actuellement, pour 170 représentants des sujets de la Fédération), affaiblit encore le Conseil de la Fédération et viole, cette fois-ci ouvertement, le principe de la séparation des pouvoirs ${ }^{38}$.

La gravité de ces atteintes portées à des principes démocratiques et, surtout, aux bases du fédéralisme amène à se demander si la Russie actuelle est toujours un État fédéral et, dans l'affirmative, quelles caractéristiques présente aujourd'hui le «fédéralisme russe».

38. On remarquera que, jusqu’à aujourd'hui, le président de la Fédération de Russie, n’a pas fait usage de ce pouvoir. Sur cette révision constitutionnelle, voir notamment, J. Demesheva, "Why Russias's Federation Cannot Become the House of Lords », Institute of Modern Russia, 19 juin 2014 https://imrussia.org/en/law/761-why-russia-s-federation-council-cann ot-become-the-house-of-lords (site consulté en juin 2018) ; A. Gazier, « Les modèles occidentaux dans les évolutions récentes du droit constitutionnel russe », in E. Zoller (dir.), Migrations constitutionnelles d'bier et d'aujourd'bui, Éditions Panthéon-Assas, p. 109 sq. 


\section{B - QUELQUES INTERROGATIONS SUR LA NATURE FÉDÉRALE \\ DE LA RUSSIE}

Comment qualifier la forme étatique de la Russie suite aux réformes centralisatrices et autoritaires menées par Vladimir Poutine? La question divise les spécialistes tant russes qu'occidentaux du fédéralisme russe. Certains évoquent une "fédération sans fédéralisme ${ }^{39}$ ", un fédéralisme «qui dépérit ${ }^{40}$ », un « fédéralisme dormant ${ }^{41}$ ", un « fédéralisme devenant fictif ${ }^{42}$ ", un «État unitaire dans un habit fédéral ${ }^{43}$ " ou encore un «post-fédéralisme ${ }^{44} »$. Cependant, la diversité de ces qualificatifs ne traduit pas de profondes différences d'appréciation. Ces spécialistes s'accordent ainsi à reconnaître que, malgré les réformes, les attributs formels de l'État fédéral russe ont été conservés. En d'autres termes, ils considèrent généralement que, au moins sur le plan du droit, la Russie demeure une Fédération. À l'appui de cette affirmation, on peut invoquer notamment le texte constitutionnel. En effet, la Constitution de 1993 a prévu, comme on l'a mentionné plus haut, une construction étatique répondant grosso modo aux grandes « lois » du fédéralisme (superposition, autonomie et participation). Certes, comme on l'a vu également, les principes d'autonomie et de participation ont été mis en cause par les politiques menées à compter de 2000 mais on peut estimer que la structure même de l'État russe n'a pas été affectée et qu'elle reste de type fédéral.

Ainsi, la Russie a toujours la forme d'un État fédéral. En revanche, son mode de gouvernement a subi des changements qui l'ont éloigné tant de l'esprit que des pratiques fédéralistes. Comme le souligne Cameron Ross, "Relations between the federal government and the regions are not based on classic federal principles of self-rule and shared rule ${ }^{45}$ ». Ces relations lui paraissent plutôt régies par les principes de hiérarchie, de centralisation et d'unité ${ }^{46}$. Reprenons un par un ces principes. La hiérarchie caractérise aujourd'hui les rapports entre le pouvoir central et les dirigeants des régions même si ces derniers ne sont pas, à proprement parler, subordonnés au président de la Russie mais parce

39. J. Radvanyi «Le fédéralisme russe : des mutations inachevées », op. cit.

40. V. Lysenko « Uvjadajuščij federalism » (Le fédéralisme qui dépérit), Žurnal "Kazanskij federalist », 2004, $\mathrm{n}^{\circ} 2(10)$.

41. A. Zaharov "O cennosti federalizma » (Sur la valeur du fédéralisme), 28/02/2017 http://ryzkov.ru/index.php/index.php ?option=com_content\&view $=$ article\&

$\mathrm{id}=37123 \quad: 2017-03-01-16-42-12 \& \mathrm{catid}=11 \quad: 2011-12-26-10-30-14 \&$ Itemid $=6 \quad$ (site consulté en mai 2018).

42. R. Sakwa, "Federalism and democracy in the Russian Federation », op. cit., p. 225.

43. C. Ross, "Federalism and defederalisation in Russia ", op. cit., p. 151

44. Shtepa (Vadim), «Kremlin Uses "Preventive Democracy" to Reinforce Russia's Post-

Federalism (Part Two)», The Jamestown Foundation, op. cit.

45. C. Ross «Federalism and defederalisation in Russia », op. cit., p. 151.

46. Idem. 
qu'il existe de véritables liens d'allégeance entre eux et le chef de l'État. La centralisation imprègne tout le système et se traduit notamment par l'octroi de pouvoirs décisionnels réduits aux régions, aussi bien dans le domaine administratif que budgétaire et fiscal ${ }^{47}$. Enfin, l'unité, qui a conduit notamment, à supprimer la différence de traitement qui existait entre les républiques et les régions ordinaires, a permis de niveler leur statut par le bas. Elle se traduit également par la puissance du parti «Russie Unie », qui joue un rôle essentiel par sa mainmise sur le pouvoir aussi bien au niveau fédéral que régional.

Ainsi, s'il y a bien un trait qui manque aujourd'hui au "fédéralisme russe », comme d'ailleurs au système politique dans son ensemble, c'est le pluralisme ${ }^{48}$. Pour autant, doit-on estimer que les dirigeants régionaux n'ont aucune autonomie, ne seraient que des courroies de transmission des volontés du centre? Une telle conclusion serait très exagérée. Sous réserve de donner des gages de loyauté à l'égard du pouvoir central, les gouverneurs disposent encore d'une marge de manœuvre non négligeable pour gérer les affaires de leur région (même si elle s'est considérablement réduite au cours des ans ${ }^{49}$. Plus précisément, les dirigeants régionaux, qui ont pour tâche essentielle tout à la fois d'assurer la stabilité politique dans leur région et de permettre au parti «Russie Unie» d'asseoir sa domination lors des élections fédérales, régionales et locales $^{50}$, continuent, par ailleurs, à jouer un rôle très important dans l'application des politiques menées, dans les domaines les plus divers, au sein de leur territoire. C'est particulièrement net dans les républiques du Caucase où, comme l'affirme Jean Radvanyi, le pouvoir central " passe une sorte d'accord avec les élites locales (les présidents et autres dirigeants officiels de chaque sujet) leur donnant carte blanche sur les plans économique et social intérieurs, à condition qu'elles assurent l'ordre établi ${ }^{51}$ ».

Ainsi, malgré la recentralisation des compétences et l'accroissement des contrôles exercés par le centre, les régions disposent d'une certaine dose de liberté, de pouvoirs tant formels qu'informels. Plusieurs républiques du Caucase du No d - Tchétchénie, Ingouchie et Daghestan peuvent même imposer, en toute impunité, des règles et des pratiques

47. Voir notamment J. Radvanyi, «Le fédéralisme russe : des mutations inachevées », op. cit.

48. Voir notamment R. Sakwa, "Federalism and democracy in the Russian Federation ", op. cit., p. 221.

49. Voir notamment R. Sakwa, ibid., p. 226 et s; A. Libman, Consequences of Informal Autonomy : The Case of Russian Federalism, Francfort sur le Main, Peter Lang, 2016, p. 167 sq.

50. Voir notamment D. Slider, "Regional governance », in Gill Graeme, Young James, eds, op. cit., p. 156-160.

51. J. Radvanyi, "Caucase : la marche turbulente de la Fédération de Russie », Hérodote, 2010/3 (n $\left.{ }^{\circ} 138\right)$, p. 20. 
violant le droit fédéral (issues notamment de la charia) ${ }^{52}$. En conséquence, on peut estimer ainsi que si le pluralisme politique est quasiment absent du fédéralisme russe actuel, il n'en est pas de même de l'autonomie des régions, qui subsiste, au moins en partie.

Au total, il apparaît que le fédéralisme russe, qui trouve son origine tout à la fois dans la politique de démocratisation menée sous la Perestroikka et dans le processus d'éclatement de l'URSS et qui a eu pour principale fonction de maintenir l'unité de la Russie, présentait dès sa formation, des traits peu conformes aux standards d'un État à la fois fédéral et démocratique. En effet, si la Russie post-soviétique s'est dotée d'une structure de type fédéral et a adopté une Constitution consacrant les principes essentiels d'une démocratie libérale, les années quatrevingt-dix ont été marquées par la montée en puissance des dirigeants régionaux et le développement, au centre comme dans les régions, de dérives autoritaires et clientélistes. À compter de l'an 2000, une politique de recentralisation autoritaire a changé la donne en rétablissant l'autorité du pouvoir central mais aussi en marquant un net recul de la démocratie et en mettant en cause le fédéralisme. Le résultat de cette évolution peut être apprécié de deux façons différentes, selon la conception que l'on retient des rapports entre fédéralisme et démocratie. Si l'on considère que ces deux notions sont inséparables, le fédéralisme russe actuel semble n'être plus qu'une coquille vide, un ensemble d'institutions «désactivées ${ }^{53}$ ». Si on dissocie le fédéralisme de la démocratie, on peut estimer que le fédéralisme russe est devenu une nouvelle forme de "fédéralisme non démocratique ${ }^{54}$ ", pouvant être qualifiée d autoritaire ou même d'autocratique ${ }^{55}$, où le gouvernement est centralisé et autoritaire tant au niveau fédéral que régional et où, malgré cela, les autorités régionales disposent d'une latitude non négligeable pour gérer leur territoire et tenir compte de ses spécificités, notamment ethniques et culturelles, - sous réserve de leur fidélité au pouvoir central.

52. Voir notamment L. Sykiainen, «Is Sharia Compatible with Contemporary Russian Law », National Research University Higher School of Economics, Working papers, Series : Law, WP BRP 49/LAW/2015 http://dx.doi.org/10.2139/ssrn.2591263 (site consulté en mai 2018).

53. Voir, sur cette «désactivation", R. Sakwa «Federalism and democracy in the Russian Federation », op. cit., p. 225.

54. Sur le «fédéralisme non démocratique », voir notamment R. Capitant, «Fédéralisme et démocratie », op. cit., p. 12.

55. Voir, à titre d'exemple, A. Obydenkova, W. Swenden, « Autocracy-sustaining versus democratic federalism : Explaining the divergent trajectories of territorial politics in Russia and Western Europe », Territory, Politics, Governance, 2013, 1 :1, p. 86-112, https://www.tandfonline.com/doi/abs/10.1080/21622671.2013.763733 Site consulté en juillet 2018. 